Portland State University

PDXScholar

2014

Affect Perception in Computer Mediated

Communication

Rachel E. Townsend

Portland State University

Follow this and additional works at: https://pdxscholar.library.pdx.edu/honorstheses

Let us know how access to this document benefits you.

Recommended Citation

Townsend, Rachel E., "Affect Perception in Computer Mediated Communication" (2014). University

Honors Theses. Paper 72.

https://doi.org/10.15760/honors.39

This Thesis is brought to you for free and open access. It has been accepted for inclusion in University Honors Theses by an authorized administrator of PDXScholar. Please contact us if we can make this document more accessible: pdxscholar@pdx.edu. 
Affect Perception in Computer Mediated Communication

by

Rachel E. Townsend

An undergraduate honors thesis submitted in partial fulfillment of the requirements for the degree of

Bachelor of Science

in

University Honors

and

Psychology

Thesis Adviser

Christopher Allen, Ph.D

Portland State University

2014 


\begin{abstract}
The perception of affect influences the subjective perception of an individual's environment (Isbell \& Burns, n.d.). Accurate affect perception leads to increased resilience and positive coping mechanisms when faced with daily life stressors (Robinson, Moeller, Buchholz, Boyd, \& Troop-Gordon, 2012). Communication technologies have revolutionized the ways in which individuals connect to one another professionally and socially (Joseph B. Walther, 1992). This study investigated accurate affect perception in computer mediated communication (CMC) from a multidisciplinary perspective. One hundred fifty four research participants $\left(M_{\text {age }}=26.84, S D_{\text {age }}=9.66\right)$ responded to a self-report questionnaire hosted by Qualtrics.com. A multiple linear regression was conducted, regressing accurate affect perception in the $\mathrm{CMC}$ environment on personality, mood, demographic information, and attitude toward CMC variables. The analysis was significant, $\mathrm{R}^{2}=.2, F(13,140)=2.60, p=.003$. and lends significant support for previous research in the cognitive neuroscience field that posits positive mood is the most significant predictor of affect perception, $\beta=.12, t(13)=2.52$, $p=.008$. Proposed methods to increase ones affect perception in CMC and future research directions are discussed.
\end{abstract}


Introduction

The perception of affect in one's environment influences an individual's subjective experience, which is significant to their objective experience (Robinson et al., 2012). Communication technologies have revolutionized the ways in which individuals connect to one another (Kelly \& Keaten, 2007). Text messages, e-mail, and IM chat programs, such as those Google and Facebook provide, allow individuals to send and receive messages on their cell phones at their convenience throughout the day. The convince communication technologies provide come with a price. The environment created by computer mediated communication $(\mathrm{CMC})$ is text based, as opposed to an environment based in social cues (Joseph B. Walther, 1992). Thus, the affect of a message received through $\mathrm{CMC}$ is more ambiguous than the affect of a message received accompanied with social cues (Perry \& Werner-Wilson, 2011).

Communication research posit several theories to accommodate the ways in which we communicate using CMC (J. B. Walther, 1996). Personality psychology research has documented the correlation between the traits extraversion and neuroticism and positive and negative affect (Larsen, n.d.). Neuroscience research posits the benefits of having positive affect (over negative affect) and what language primers cognitively bias an individual towards an emotion (Robinson et al., 2012) (Van Berkum, De Goede, Van Alphen, Mulder, \& Kerstholt, 2013). The present study investigated accurate affect perception in computer mediated communication (CMC) from a multidisciplinary perspective. The purpose of the present study was to investigate 
accurate affect perception in the CMC environment and under which conditions affect perception increases.

Lit Review

Affect

Affect is described as the positive or negative feeling and reactions one experiences toward any given stimulus (Isbell \& Burns, n.d.). Affect perception is defined as how an individual perceives a stimulus (Robinson et al., 2012). The way in which an individual perceives a stimulus shapes the way in which the individual responds to the stimulus. Affect perception plays an influential role in social interactions, decision making processes, and the way in which one interoperates their environment (Isbell \& Burns, n.d.).

Individuals who are high in accurate affect perception are found to be less reactive to daily life stressors than individuals who are low in accurate affect perception (Robinson et al., 2012). Further, the individuals who are high in accurate affect perception react to stressors in less adverse manners than those who are low in affect perception. Robinson's research indicated that affect perception may act as a social and psychological buffer (Robinson et al., 2012). The research states that everyone has stressors and becomes depressed; however individuals rated high in affect perception recover from depression and are more resilient than individuals who are low in affect perception (Robinson et al., 2012). 
There is a clear difference between affect, emotional response and cognition. Jaak Panksepp, an affective neuroscientist, argues that the order in which humans process a stimulus is affect, then emotional response and cognitions second (Panksepp, 2003). According to Panksepp, affect takes place in the subcortical regions of the brain (limbic system, cingulate gyrus) and is a cross-cultural experience (Burgdorf \& Panksepp, 2006). Panksepp defines affect as an emotional filter of positive or negative evaluation of the stimulus (Panksepp, 2003). The affective filter is so powerful that there can be no biological brain and philosophical mind separation (Panksepp, 2003).

Prior research has established a connection between cognition and emotional response (Brown \& Taylor, 1986) (Ochsner \& Gross, 2005). Emotion theory posits emotions are responses to external or internal stimuli and/or are mental representations that involve changes across multiple response systems (behavioral, experiential, peripheral physiological) (Ochsner \& Gross, 2005). Emotions have an identifiable object or trigger, can either be unlearned or unlearned responses to stimuli with intrinsic affective properties or with acquired emotional value, and may involve various types of appraisal processes that asses the significance of the stimuli to the current goal that depend on the neural system (Ochsner \& Gross, 2005).

Affective response, the cross-cultural filter in which every individual perceives a stimulus, takes place in the brain's subcortical structures (Burgdorf \& Panksepp, 2006). Emotional responses and cognitions are higher cognitive functions framed by an individual's experience and environment (Panksepp, 2003). Emotional regulation can be controlled through cognition and stimulus-response conditioning (Ochsner \& Gross, 
2005). The brain's malleability can reprogram how an individual interoperates a stimulus by learning to associate a new emotional response to the stimulus (Ochsner \& Gross, 2005). Forming new neuroconnections, or pathways between neurons, is called neuroplasticity (Reisberg, 2012). When neurons fire together, pathways are formed and strengthened (Reisberg, 2012). Cognitive appraisal therapies which condition the brain in a specific response to a stimulus are highly quantifiable and show the neuroplasticity of the brain (Ochsner \& Gross, 2005) (Hölzel et al., 2011).

For the purposes of this paper, we use Panksepp's argument that the affective and cognitive consciousness are distinctly organized in the brain, with affect arising directly from subcortical regions where the executive systems for emotional responses are organized (Panksepp, 2003). Higher cognitive regions of the brain give individuals a rich experience of their emotions (emotional response) and allow for complex problem solving (cognitive function) (Panksepp, 2003).

Personality and Affect Perception

Personality is described as an enduring pattern of attributes, cognitions, and behavior that is relatively constant over time and across situations (Löckenhoff \& Costa, n.d.). Personality traits describe the general dimensions in the differences among individuals. The Big Five or the Five-Factor Model is the conceptual framework in which the different dimensions are organized (Löckenhoff \& Costa, n.d.). 
The Five Factor personality traits are Openness to experience (sometimes called Intellect), Conscientiousness, Extraversion, Agreeableness, and Neuroticism. Briefly, Openness/Intellect is described as creative, imaginative, curious, and comfortable with a diversity of emotions; Conscientiousness is described as hard working, organized, determined, and reliable; Extraversion is described as warm, cheerful, sociable, and assertive; Agreeableness is described as helpful, trusting, altruistic, and avoid confrontation; and Neuroticism is described as anxious, prone to experience negative emotions, and self-conscious (Löckenhoff \& Costa, n.d.). The Big Five are cross-cultural traits (Löckenhoff \& Costa, n.d.).

The five factor traits are scale scores with polar ends (Löckenhoff \& Costa, n.d.). Everyone places somewhere on each of the five factors. Once adulthood is reached, traits become relatively stable (Cobb-Clark \& Schurer, 2012). Research has shown malleability in adulthood to be a consequence of major life changes and challenges (Cobb-Clark \& Schurer, 2012). Trait changes at the statistically significant level occur for men and women when experiencing employment and income related challenges; while family and life related events are statistically insignificant to trait change (Cobb-Clark \& Schurer, 2012).

Personality theory posits the traits extraversion and neuroticism are the two dominant traits when perceiving affect and communicating (Eysenck, 1967) (Jeffrey A. Gray, 1970). Extraversion and neuroticism are two most researched traits due to their predominance nature in the individual and occurrence in every culture (Löckenhoff \& Costa, n.d.). 
In the broadest sense, the personality trait extraversion is the extent to which someone enjoys social interactions (Lucas, n.d.). Extraversion is generally described as outgoing, enjoy the company of others, talkative, active, and cheerful (Löckenhoff \& Costa, n.d.). The personality trait neuroticism is generally described as how an individual experiences their environment to be threatening, dissatisfactory, and unsafe (Kwon \& Weed, n.d.). Those who are high on the neurotic scale are prone to experience a more frequent and greater amount of anxiety then those who are low on the neurotic scale (Kwon \& Weed, n.d.).

Eysneck's biological personality theory posits that the ascending reticular activation system as the cause of an individual's arousal baseline level (Eysenck, 1967). Eysneck postulated that extraverts have lower levels of arousal compared to introverts, thus they seek arousal through external sources (Eysenck, 1967). Gray's Biopsychological Theory of Personality expands on Eysneck's theory by introducing the concept of the behavioral activation system (BAS) and behavioral inhibition system (BIS) (J. A. Gray, 1981).

The BAS and BIS offer theatrical rationale to predicting the emotional susceptibility of extraverts and neurotics. Gray posits that the two systems are neurotically based motivators that result in explaining a majority of the observed behavioral and emotional differences between extraverts and neurotics (J. A. Gray, 1981). The BAS is thought to regulate reward signals and the BIS is thought to regulate punishment signals (J. A. Gray, 1981). 
Gray's Biopsychological Theory of Personality is one of the most widely research and accepted personality theories. Research has found that extraversion is sensitive to reward signals; thus it correlates with the BAS system and positive affect (Larsen \& Ketelaar, 1991). Neuroticism is sensitive to punishment signals; thus neuroticism correlates with the BIS system and negative affect(Larsen \& Ketelaar, 1991). Sensitivity to reward and punishment signals are the major determinates for individual coping strategies, understanding the other's perspective, seeking support, and presence during problem solving (i.e. withdrawal from situation vs actively engage in problem solving) (Lee-Baggley et al., 2005).

Personality theory suggests that affect perception is hardwired into the brain. The correlation between the trait extraversion and positive affect is well established in research (Lucas, n.d.). Prior research found that different levels of extraversion have significantly different responses to the same environmental stimulus (Larsen \& Ketelaar, 1989). While under mood induction, high extraverts are less reactive to negative stimulus and more reactive to positive stimulus; while low extraverts are reactive to negative stimulus and positive stimulus (Larsen \& Ketelaar, 1989). However; low extraverts are not as reactive to positive stimulus as high extraverts (Larsen \& Ketelaar, 1989). Further, extraverts are more reactive to positive stimulus than ambiverts individuals who are in the middle of the introvert and extrovert scale (Yuan et al., 2012). Ambiverts display traits of an extravert at times and traits of an introvert at times; thus they are neither extreme, but walk the middle path (Yuan et al., 2012). Thus, individuals 
with a highly active BAS seek reward in stimulus and either find the reward or they create the reward.

The correlation between the trait neuroticism and negative affect is well established in research (Suls \& Martin, 2005). Prior research has found that neurotics are sensitive to negative stimulus (Larsen \& Ketelaar, 1989)(Larsen \& Ketelaar, 1991). While under mood induction, high neurotics are more reactive to negative stimulus and less reactive to positive stimulus, while low neurotics are not as reactive to negative stimulus as high neurotics (Larsen \& Ketelaar, 1989). Neurotics attend to negative stimulus selectively, perceive it to a higher severity, and recall it with higher accuracy than those who are low on the neurotic scale (Suls \& Martin, 2005). High neurotics carry poor moods forward, create conflict where none existed, seek approval, and question others affection towards them, thus creating a cascade of emotion that may lead to poor decision making and punishment (Suls \& Martin, 2005). Thus, an individual with a highly active BIS system seeks punishment and either finds punishment or creates punishment.

In summary, Grays's Biopsylological Theory suggests that extraverts seek reward and find reward, while neurotics seek punishment and find punishment; thus, extraverts have a more sensitive BAS system and neurotics have a more sensitive BIS system (J. A. Gray, 1981). Extraverts and neurotics are hard wired to perceive the world in opposite ways (Howell \& Rodzon, 2011). Prior research suggests that extraverts and neurotics will correlate with positive and negative affect regardless of mood and environment (Uziel, 2006). The purpose of the present research is to better understand the role of 
personality and affect perception in the CMC environment. To our knowledge, no prior research has measured personality traits and affect perception in the CMC environment.

Computer Mediated Communication (CMC) and Affect

Spitzberg (2006) defined CMC as, "any human symbolic text-based interaction conducted or facilitated through digitally based technologies...that requires actual people engaged in a process of message interchange in which a medium of exchange at some point is computerized" (Spitzberg, 2006, pp. 630-631). For the purposes of the present study, CMC refers to e-mail, text messages without emoticons (smiley faces), and instant messages.

The increasing forms and frequency of CMC use in today's society has created an omnipresence of constant interpersonal communication that transcends borders, time zones, class, ethnicities, and gender (Coyne, Stockdale, Busby, Iverson, \& Grant, 2011)(Bergdall et al., 2012). The economic burden of owning a cell phone and internet service has rapidly declined over the years (Odlyzko, 2012). The socioeconomic gap is rapidly closing between those who grow up tech-savvy with technology in the home and those who become tech-savvy later in life when they are able to obtain technology (Bergdall et al., 2012). 
CMC research posits those born before 1980 (tech-immigrants) versus those born in 1980 and after (tech-natives) have different attitudes toward CMC based from their perceptions of CMC (Bergdall et al., 2012). Research posits that tech-natives, having grown up in a world where technology is present and always changing and have integrated CMC into a greater number of daily communications than tech-immigrants (Coyne et al., 2011). Tech-natives are more likely to capitalize on the benefits of CMC, understand how to manipulate the medium to their advantage, and possess greater amounts of positive associations with communicating through CMC versus techimmigrants (Coyne et al., 2011). However, a majority of the $\mathrm{CMC}$ research, and the theory based off the research, has been conducted with college-aged students who are tech-natives.

Communication research reports several positive aspects to $\mathrm{CMC}$ such as $\mathrm{CMC}$ may help reaffirm boundaries, assists in maintaining bonds, keeping a record of interactions, and allowing for carefully thought out replies (Ganong, Coleman, Feistman, Jamison, \& Stafford Markham, 2012). CMC assists people in feeling emotionally close to loved ones when they cannot be geographically close (Coyne et al., 2011). Further, CMC connects individuals to one another in ways that individuals could not be connected a generation ago (Bergdall et al., 2012).

Researchers in communication are quick to point out that $\mathrm{CMC}$ is not the perfect communication medium. CMC is an asynchronous form of communicating, thus may not be always appropriate (Tong \& Walther, 2012). Contextually, asynchronous communication may be seen as problematic for a user (Topi, Valacich, \& Rao, 2002). 
Using CMC to resolve conflicts may take more time and higher levels of conflict are perceived when compared to face to face interactions (Topi et al., 2002). Further, the lack of social cues in CMC may be seen as a problematic when dealing with complex or highly emotional interactions (Byron, 2008). CMC may also be used as a means of gaining control over another by ignoring messages or omitting relevant information (Ganong et al., 2012).

$\mathrm{CMC}$ is the medium in which the message is communicated. Accurate interpretation of the message is dependent on the receiver and the message content. One of the most widely accepted interpersonal dynamic theories that explain online behavior is Walther's Hyperpersonal Model of CMC (J. B. Walther, 1996). The Hyperpersonal model posits that in an environment that lacks social cues such as facial expression, body posture, and tone of voice, one will not experience depersonalization. Instead, the individual may capitalize on the medium's lack of social cues by editing messages more deliberately than if they were conversing in a face to face environment, which gives them heightened control over their self-presentation (J. B. Walther, 1996). In other words, the greater syntactic command one has over their language, the better their self-presentation through CMC and message interpretation abilities (Tong \& Walther, 2012). Research posits the Hyperpersonal model can help explain email sender's likability (Byron \& Baldridge, 2007).

Walther's Social Information Processing Theory (SIP) posits, in the absence of social cues, an individual creates cues in text which convey their intended affect in CMC is through punctuation, capitalization and grammar (Joseph B. Walther, 1992). Life 
experience and education increase an individual's ability to read, write and intemperate a language (Tong \& Walther, 2012).

The purpose of this study was to investigate accurate affect perception in the CMC environment and under which conditions accurate affect perception increases. According to $\mathrm{CMC}$ research, those conditions would be a positive attitude toward $\mathrm{CMC}$ formed either through experience or age along with the learned ability to manipulate and read language.

Mood and Affect

Cognitive neuroscience posits that mood and affect are interrelated but not the same (Panksepp, 2003). Affect is hardwired into subcortical regions of the brain, mood is a result of higher cortical functions (Burgdorf \& Panksepp, 2006).

Mood is a relatively mild state and that is long lasting, it has no identifiable object or trigger (Cohen-Charash \& Boyd, 2007). Mood is always present, though we are not always aware of it (Cohen-Charash \& Boyd, 2007). Moods are generally positive or negative, although they are often conflated with an emotional response to a stimulus when being spoken about (Cohen-Charash \& Boyd, 2007). In other words, a mood casts a tone to an individual's experience. Mood and affect are interrelated with stimulus filtering functions; however their differences are important to remember. 
Neuroscience and linguistic research indicate syntactic complexity and text comprehension negatively impacted the mood of students in an academic environment (Bohn-Gettler \& Rapp, 2011). This study indicated that mood significantly impacted reader's comprehension and memory (Bohn-Gettler \& Rapp, 2011).

Neuroscience and linguistics posit that mood creates a language processing parameter (Van Berkum et al., 2013). As one reads or listens to a conversation, they anticipate the next word. This phenomena is called using cognitive heuristics and syntactic parsing (Reisberg, 2012). However, a poor mood will disinhibit the ability to use these methods (Van Berkum et al., 2013). Further, individuals who are in a positive mood have been shown to possess an increased capability of making intuitive judgments and detect sematic coherence with greater accuracy than those in a neutral moods (Sweklej, Balas, Pochwatko, \& Godlewska, 2014).

Context assist in giving ambiguous stimulus, such as words in text, boundaries. The Circumplex Model of Affectivity uses a multidimensional scale to rate affect as either being positive or negative and high or low arousal (Russell, 1980). The positivenegative scale is placed on the vertical axis, the high-low arousal scale is on the horizontal axis, and the neutral point is where the two axis intersect (Russell, 1980). This model gives a visual representation of the cognitive process categorization of a stimulus. When research participants placed 28 emotion words such as happy, delighted, morose, angry, sad, and tired on the multidimensional scale, researchers found that words lack sharp boundaries (Russell, 1980). In other words, a single word may be assigned to more than one position on the scale. 
The various placement of a single word is due to the malleable nature of language. Gradual transitions from one category to another are dependent on the context surrounding the word's use and an individual's schema for the word. Thus, affective words tend to have an circular ordering in a cognitive affective space (Russell, 1980). The overlapping circular order of affect means affective states are interdependent (as opposed to separate) on one another (Russell, 1980).

The Affect Infusion Model (AIM) is a widely accepted dynamic theory that accounts for many ways in which an individual arrives at a judgment. It posits an individual bases social judgments on direct access, motivation, heuristic, and substantive processing (Forgas, 1995). Direct access is used when retrieving a preexisting judgment, motivation is used when computation of a judgment is guided by a specific motivation, heuristic is used when one wishes to construct a judgment using a shortcut, and substantive processing is used when one must engage in selective constructive judgment based on available information and previous experience (Forgas, 1995). Further, the AIM states the degree in which one infuses affect into a situation depends on the judgment strategy chosen. The strategies with higher affect infusion are heuristic and substantive processing methods (Forgas, 1995). Due to the nature of the CMC environment's lack of social cues combined with the results of Van Berkum's results regarding language processing, the use of heuristic judgment may be a common way to form opinions and judgments when communicating.

The purpose of this study was to investigate accurate affect in the CMC environment and under what variables increase one's ability to read affect. According to 
research and theories on the effects of mood on affect and their relationship with syntax, the significant variable would be the level of positive or negative mood because mood is a filter that impacts higher cognitive functioning.

Hypothesis

The purpose of this research is to understand the variables -together and uniquely - that assist in accurate affect perception in the $\mathrm{CMC}$ environment. Personality research strongly correlates the traits extraversion and neuroticism with positive and negative affect (J. A. Gray, 1981); but to our knowledge has not investigated these traits and the ability to accurately interoperate affect in the CMC environment. Communication research has investigated the use and under what conditions the comprehension of the reader may increase (Joseph B. Walther, Deandrea, \& Tong, 2010); but to our knowledge, has not applied the understanding to personality research on affect perception or the mood variable. Neuroscience and linguistic research has investigated the relationship between affect and mood (Brown \& Taylor, 1986), affect and cognition (Panksepp, 2003), and the way mood effects the cognitive process (Van Berkum et al., 2013); however, they have not investigated any of these variables in the environment of $\mathrm{CMC}$. 
There are strong correlations between the trait extraversion and positive affect (Larsen \& Ketelaar, 1989)(Larsen \& Ketelaar, 1991). Further, extraversion, being socially savvy, pay more attention to the external environment (compared to introverts), thus have increased ability to decode social situations (Lieberman \& Rosenthal, 2001). What is not known is if high extraverts are able to decode affect in the CMC environment. Thus, the present study hypnotizes:

H1a: As the level of an individual's extraversion increases, their ability to accurately perceive affect in the CMC environment will significantly increase.

There are strong correlations between personality trait neuroticism and negative affect (Larsen \& Ketelaar, 1989). Neurotics are known to possess maladaptive coping mechanisms for daily stressors that leads to a negative subjective perception of the world (Suls \& Martin, 2005). What isn't fully understood is how the neurotic's perception relates to accuracy in affect perception in the $\mathrm{CMC}$ environment. Thus, the present study hypothesizes:

H1b: As the level of an individual's neuroticism increases, their ability to accurate perceive affect in the CMC environment will significantly decrease.

CMC research states that those born before 1980 (tech immigrants) versus those born in 1980 and after (tech natives) have different perceptions of CMC (Bergdall et al., 2012). Age is the most cited underlying variable for an individual's ability to accurately 
perceive messages in the CMC environment (Bergdall et al., 2012). The individual's age impacts their amount of daily technology use and level of education (Bergdall et al., 2012)(Coyne et al., 2011) (Joseph B. Walther et al., 2010). What isn't fully understood is the relationship of these demographic variables with accuracy in interpreting the sender's message. Thus the present study hypothesizes:

H2a: As the age of the individual increases, their ability to accurately perceive affect in the CMC environment will significantly increase.

$\mathrm{H} 2 \mathrm{~b}$ : As an individual increases their daily $\mathrm{CMC}$ use, their ability to accurately perceive affect in the CMC environment will significantly increase.

$\mathrm{H} 2 \mathrm{c}$ : As the individual's education level increases, their ability to accurately perceive affect in the CMC environment will significantly increase.

Previous research has associated mood with ability to comprehend and make judgments on language (Van Berkum et al., 2013). What isn't fully understood is the relationship between the positive or negative mood of a message receiver and their accuracy in perceiving the message sender's affect in the CMC environment. Thus, the present study hypothesizes:

H3a: As the level of positive mood increases, the ability to accurately perceive affect in the $\mathrm{CMC}$ environment will significantly increase. 
$\mathrm{H} 3 \mathrm{~b}$ : As the level of negative mood increases, the ability to accurately perceive affect in the CMC environment will significantly decrease.

\author{
Methods
}

Participants

A questionnaire was made available online through Qualtrics.com. The link to the study was distributed via URL. Two hundred thirteen individuals responded to the questionnaire. One hundred fifty four participants $\left(M_{\text {age }}=26.84, S D_{\text {age }}=9.66\right)$ completed the questionnaire. Their ages ranged from 18 to 64 years. There were no incentives given to participants for taking part in this study.

Participants were primarily recruited via a URL link distributed to students through the Portland State University Honors College list serve and posters on Portland State University's campus. Secondary sources were recruited by posters on Portland Community College campuses and a Polyamory Weekly podcast announcement and link on website the website. Source analysis revealed no statistically significant difference between sources.

Procedure

Participants used the URL link to go to Qualtrics where the study's survey was hosted. Informed consent was on the first page of the survey and by clicking the next 
button, the participant gave consent. Participants answered the survey in the following order: demographic questions, the Mini IP-IP, the ACCS, the PANAS, and affect perception questions.

The duration of data collection was six week during January and February of 2014.

Measures

CMC Competence and Use. Demographics included age, education level, and daily use. According to research, age, education level, and daily use are the three most significant variables in determining an individual's ability to use and manipulate CMC (Coyne et al., 2011) (Bergdall et al., 2012).

Participants entered their age into a blank text box. Participants selected their education level from the following options: 1 = High School, 2 = Some College, 3 = Associates Degree, 3 = Bachelor's Degree, $5=$ Graduate Degree, and $0=$ Other .

Participants chose their daily use from a drop down list. The option the participants chose from were as follows: $1=0$ or no messages, $2=1-25$ messages, $3=$ 26-50 messages, 4 = 51-99 messages, 5 = 100-199 messages, $6=$ over 200 messages .

Personality measure. The Mini IP-IP is a 20 item self-report brief personality measure that measures the Big-5 personality domains (Intellect, Conscientiousness, 
Extraversion, Agreeableness, and Neurotic) (Donnellan, Frederick, Oswald, \& Lucas, 2006). The Mini IP-IP uses the word Intellect in the place of Openness. The items are phrases (Ex: "Am the life of the party", "Make a mess of things") which the participant is asked to indicate how well each phrase describes them.

Each of the five facets of personality have four questions which are scored by summing them together on a Likert scale from one to five (one being strongly disagree, three being neither disagree or agree, and five being strongly agree). This gives each of the personality facets their own score.

Previous research reported mean scores for adults $(\mathrm{N}=1,481, \mathrm{M}=24.5, \mathrm{SD}=$ 8.73) in Extraversion $(\mathrm{M}=12.99, \mathrm{SD}=3.83, \alpha=.81)$, Agreeableness $(\mathrm{M}=16.57, \mathrm{SD}=$ 2.85, $\alpha=.7)$, Conscientiousness ( $M=13.22, S D=3.53, \alpha=.68)$, Neuroticism ( $M=11.81$, $S D=3.72, \alpha=.72)$, and Intellect $(M=15.81, S D=3.11, \alpha=.7)$ using the Mini IP-IP scale (Cooper, Smillie, \& Corr, 2010). Research has shown a strong correlation between personality traits and ability to read, understand, and interoperate affect; thus, the Mini IP-IP was included in the questionnaire (Larsen \& Ketelaar, 1991). The goal of the present research is to understand if personality trait research on accurate affect perception holds true in the environment of CMC.

Mood measure. The PANAS is a 20 item self-repot measure of mood (Watson, Clark, \& Tellegen, 1988). The scale has two halves: ten questions to measure the participant's level of positive affect (PA) and ten question to measure the participant's 
negative affect (NA). The PANAS is a highly reliable measure (PA $\alpha=.89$; NA $\alpha=.85$ )

(Crawford, 2004). Previously reported mean scores for PANAS $(N=1,003)$ are PA $(M=$ 31.31, SD = 7.65) and NA $(M=16, S D=5.9)$ (Crawford, 2004).

The PANAS was included in this questionnaire in order to understand the emotional state of the participant at the time of taking part in the study. Participants were asked to answer the affective words (excited, jittery, interested, alert, etc.) based on a Likert scale from one to five (one being very slightly/not at all, three being moderately and five being extremely) and given the time frame of how often they have felt that way over the past 48 hours. The goal of the present research is to understand how mood effects accurate perception in the environment of CMC.

Attitude Toward CMC. The Affect for Communication Channels Scale (ACCS) is a self-report 21 item measure (Kelly \& Keaten, 2007). The ACCS measures positive and negative feelings associated with communicating via CMC and face to face. The 21 items are broken into three groups on a five point Likert scale with seven statements (I enjoy e-mail because I have more time to organize my ideas; I like face to face because you can see the other person's reactions; when I use e-mail I say things that I wouldn't say face to face; etc.) per group.

The first measure is for increased preparation and control (with CMC) (IPC). The second measure is for enhanced meaning and emotion (with face to face) (EME). The third measure is for reduced anxiety and inhibition (with CMC) (RAI). For the purposes of this study, EME was reversed scored so that all of the scores would be one way 
(positive direction) toward CMC. Scores for each group were summed to create a score for each group. Previous research reported mean scores for IPC (N=630, $M=23, S D=$ 5.25, $\alpha=.92)$, EME $(N=636, M=29.5, S D=4, \alpha=.84)$ and RAI $(N=630, M=18.5, S D=$

$4.7, \alpha=.8$ ) over the course of two studies (Kelly \& Keaten, 2007). The ACCS was included in the present research to gain insight into participant's attitudes toward CMC which may effect and individual's CMC experience, thus affect perception.

Affect Perception. Nine common neutral words or statements (whatever, we'll see, hmmm..., let me think about it, I don't think so, I'm busy, sure, let's talk, fine) that participants rated on a Likert scale from one to five. One being bad/negative, three being a neutral point, and five being good/positive. The scores from each of the nine items were summed together to make the affect accuracy score with twenty-seven being a completely neutral score. The affect perception in CMC environment score was used as the dependent variable in the study.

\section{Design Overview}

A multiple linear regression was conducted regressing accurate affect perception in the CMC environment against demographics, Big-5 personality scores, ACCS scores (IPC, EME and RAI), and PANAS scores (PA and NA). 


\section{Equipment}

Data was analyzed using SPSS on a Windows Surface Pro.

\section{Results}

A multiple linear regression analysis was conducted, regressing accurate affect perception in CMC environment on participant age, education level, the participant's daily use, PANAS NA score, PANAS PA score, Extraversion score, Agreeableness score, Conscientiousness score, Neuroticism score, Intellect score, ACCS IPC score, ACCS RAI score, and ACCS EME score. Table 1 provides the results of the multiple regression analysis. Together, the predictor variables accounted for a moderate proportion of the variance in accurate affect perception in the CMC environment, $R^{2}=.2, F(13,140)=$ $2.60, p=.003$.

Select demographic questions were statically significant. Controlling for the other predictor variables, age is statically significant and positively related to accurate affect perception in the CMC environment, $\beta=.7, t(13)=1.94, p=.05$. Controlling for the other predictor variables, education level was statically non-significant and positively related to accurate affect in the $C M C$ environment, $\beta=.48, t(13)=1.49, p$ $=.14$. Controlling for the other predictor variables, daily use was negatively related to accurate affect perception in the $\mathrm{CMC}$ environment, $\beta=-.14, \mathrm{t}(13)=-.58, \mathrm{p}=.56$. 
An individual's mood and accurate affect perception had statically significant results. Controlling for the other predictor variables, the PANAS Positive Affect score was significantly related to accurate affect perception in the CMC environment, $\beta=.12$, $t(13)=2.68, p=.008$. Conversely, controlling for the other predictor variables, the PANAS Negative Affect score was negatively related to accurate affect perception in the $\mathrm{CMC}$ environment at a non-significant level, $\beta=-.04, \mathrm{t}(13)=-.87, \mathrm{p}=.39$.

Attitude towards $\mathrm{CMC}$ use was not a statically significant predictor of accurate affect perception in the CMC environment. Controlling for the other predictor variables, the ACCS IPC score was positively related to accurate affect perception, $\beta=.07, t(13)=$ 1.83, $p=.07$. Controlling for the other predictor variables, the ACCS RAI score was negatively related to accurate affect perception in $C M C, \beta=-.04, t(13)=-1.01, p=.31$. Controlling for the other predictor variables, the ACCS EME score was negatively related to accurate affect perception in $C M C, \beta=-.05, t(13)=-.98, p=.33$.

Personality was not a statically significant predictor variable. Controlling for the other predictor variables, Extraversion was positively related to accurate affect perception in the $C M C$ environment, $\beta=.29, t(13)=1.43, p=.15$. Controlling for the other predictor variables, Agreeableness was negatively related to accurate affect perception in the $\mathrm{CMC}$ environment, $\beta=-.15, \mathrm{t}(13)=-.77, p=.46$. Controlling for the other predictor variables, Conscientiousness was negatively related to accurate affect perception in the $\mathrm{CMC}$ environment, $\beta=-.02, \mathrm{t}(13)=-.12, \mathrm{p}=.92$. Controlling for the other predictor variables, Neuroticism was negatively related to accurate affect perception in the $\mathrm{CMC}$ environment, $\beta=-.06, \mathrm{t}(13)=-.31, \mathrm{p}=.76$. Controlling for the 
other predictor variables, Intellect was negatively related to accurate affect perception in the CMC environment, $\beta=-.07, t(13)=-.33, p=.74$.

Table 1. Results of Multiple Linear Regression on Accurate Affect Perception in the CMC Environment on Participant Age, Education Level, Daily Use, PANAS NA Score, PANAS PA Score, Extraversion Score, Agreeableness Score, Conscientiousness Score, Neuroticism Score, Intellect Score, ACCS IPC Score, ACCS RAI Score, and ACCS EME Score.

\begin{tabular}{lccc}
\hline & $\beta$ & $\mathrm{SE}(\beta)$ & $\mathrm{t}$ \\
\hline Intercept & 19.20 & 5.00 & $3.84^{*}$ \\
Age & 0.70 & 0.04 & $1.94^{*}$ \\
Education Level & 0.48 & 0.32 & 1.49 \\
Daily Use & -0.14 & 0.25 & -0.58 \\
PANAS Negative Affect & -0.04 & 0.05 & -0.87 \\
PANAS Positive Affect & 0.12 & 0.05 & $2.68^{*}$ \\
Extraversion & 0.29 & 0.21 & 1.43 \\
Agreeableness & -0.15 & 0.20 & -0.77 \\
Conscientiousness & -0.02 & 0.19 & -0.12 \\
Neuroticism & -0.06 & 0.21 & -0.31 \\
Intellect & -0.07 & 0.20 & -0.33 \\
ACCS IPC & 0.07 & 0.04 & 1.83 \\
ACCS RAI & -0.04 & 0.04 & -1.01 \\
ACCS EME & -0.05 & 0.05 & -0.98 \\
\hline
\end{tabular}

Note: ${ }^{*} \mathrm{p}<.05 . \mathrm{N}=154$. Model $\mathrm{R}^{2}=.2, \mathrm{~F}(13,140)=2.6, p=.003$.

\section{Discussion}

The purpose of this research was to better understand what variables inhibit the ability to accurately decode affect in the CMC environment. A multiple linear regression was conducted regressing the participant's accurate affect perception score on the predictor variables (demographic information, personality, attitude toward CMC, and mood). The model was significant at $p=.003$. Multiple regression revealed that the significant predictor variables of accurate affect perception in the CMC environment are 
level of positive mood $(p=.008)$ and the age of the individual $(p=.05)$. The analysis revealed that the ACCP IPC score $(p=.07)$ was trending toward significance. The results indicate that as an individual's positive mood increases and age increases, so does their ability to accurately perceive the affect in a CMC message. Further, the data indicates that an increase in positive feelings towards the ability to think about messages and prepare responses while using CMC to communicate may assist in accurately interoperating affect in the $\mathrm{CMC}$ environment.

The present research's finding that positive mood is a significant predictor of accurate affect perception in the CMC environment support and expand Forgas AIM model, Walther's Hyperpersonal, and Walther's SIP theory (Forgas, 1995)(J. B. Walther, 1996)(Joseph B. Walther, 1992). The AIM model posits affect infusion is the process in which the affectively loaded information exerts influence upon and becomes incorporated into the judgment process (Forgas, 1995). Our research significantly showed that positive mood effected how an individual perceived a neutral stimulus in the CMC environment. Walther's Hyperpersonal model of communication postulates that in an environment that lacks social cues, rather than experiencing depersonalization, they capitalize on the positive aspects of the channel of communication by constructing and editing messages for increased control over selfpresentation (J. B. Walther, 1996). Walther's SIP theory says that as non-verbal cues go down, language based context cues must go up (Joseph B. Walther, 1992) In the environment of $\mathrm{CMC}$, affect perception is void of social cues, thus the individual must rely on cognitive heuristics to interoperate a stimulus in the environment. Furthermore, 
the Circumplex Model of Affectivity says that words are malleable and cognitions can give the words boundaries (Russell, 1980). Mood primes cognitive heuristics (Van Berkum et al., 2013). Our finding that positive mood effects accurate affect perception furthers cognitive-linguistic research (Van Berkum et al., 2013).

It was not surprising that our research found age to be a significant predictor of accurate affect perception in the $\mathrm{CMC}$ environment. $\mathrm{CMC}$ research posits age as the biggest indicator of whether or not an individual uses technology and has had the time to acquire knowledge and syntactic command over language (Antheunis, Schouten, Valkenburg, \& Peter, 2011). Further, previous research indicates that as we age, we increase in cognitive control of emotion and decrease in reacting to affect reasponses (Panksepp, 2003).

The mean age of the present study was 26.84 years old; which is about seven years younger than individuals born in 1980 and about the time the brain's prefrontal cortex fully matures (Panksepp, 2003). These individuals have had the privilege of growing up as tech-natives with enough time to gain life experience and cognitive control of their emotional reactions (when compare to younger participants) (Bergdall et al., 2012) (Ochsner \& Gross, 2005). Further, this group has the privilege of being sandwiched between tech-immigrants who typically do not use CMC short had or emoticons and younger tech-natives (Bergdall et al., 2012). Thus, the mean age for the present study enjoy optimal self-presentation and syntactic manipulation skills to both groups, which may have resulted in an over-all positive attitude toward CMC (ACCE IPC $p=.07)$. A larger sample size is needed in order to better understand the finding. 
This study focused on a breadth of indicators of accurate affect perception in the CMC environment. The findings of this study are not generalizable due to the sample. Future research should increase their sample and focus on depth - specific indicators, as opposed to general categorical indicators.

Personality and Accurate Affect Perception

Personality research indicates extraversion and neuroticism are highly correlated with positive affect and negative affect in face to face social interactions (J. A. Gray, 1981)(Larsen \& Ketelaar, 1989). The purpose of the present research is to gain further insight in the role of personality in interpreting affect in the $\mathrm{CMC}$ environment. Results for Hypothesis 1a and 1b were inconclusive. The multiple linear regression analysis revealed that the personality trait extraversion was positively related to accurate affect perception in the $\mathrm{CMC}$ environment at the significance level of $p=.15$ and the personality trait neuroticism was negatively related to accurate affect perception in the CMC environment at the significance level of $p=.76$.

Although the present study's results were inconclusive, the relationship between personality traits and affect perception in the $\mathrm{CMC}$ environment were as expected and in line with Gray's Biopsylogical Personality Theory (J. A. Gray, 1981). Extraversion was positively related to accurate affect perception, meaning the more extraverted one was, 
the more accurate their affect perception was. Neuroticism was negatively related to accurate affect perception in the $\mathrm{CMC}$ environment.

The results could be a result of measure, stimulus or sample size. The Mini-IPIP is a reliable and brief measure for personality in questionnaires (Donnellan et al., 2006); but a more in depth questionnaire may have been more appropriate (the tradeoff being a larger dropout rate). The current research showed trends that upholds previous personality research posits extraverts and neurotics perceive the same stimulus in different ways (Larsen \& Ketelaar, 1989)(Suls \& Martin, 2005)(Uziel, 2006). Previous research found that extraverts were not significantly more reactive to neutral stimulus (when compared to introverts) unless the stimulus had high reward value (Lucas \& Baird, 2004). Participants were anonymous volunteers who took the survey online; thus they may not of placed enough value in the outcome of the study.

Further research in on the relationship between personality and affect perception in the CMC environment should be conducted focusing on level of extraversion and neuroticism, ambiguous words, and relationship depth/type. For example, a High Neurotic in a 20 year domestic partnership who is arguing with their partner over domestic chores will most likely interoperate the text message, "Hmmm..." much differently than a High Neurotic who is in their first year of a domestic partnership, arguing over domestic chores, and receives the same text message.

Demographic and Accurate Affect Perception 
CMC research states that those born before 1980 (tech immigrants) versus those born in 1980 and after (tech natives) have different perceptions of CMC (Bergdall et al., 2012). According to CMC research, age is the most accurate predictor of ability to use $\mathrm{CMC}$ and perceive messages with education and competence of use falling second and third (Bergdall et al., 2012)(Coyne et al., 2011) (Joseph B. Walther et al., 2010). The goal of the present study is to better understand the relationship between an individual's age, education, and daily use with accurate affect perception in the CMC environment.

The multiple linear regression analysis revealed age was positively related to accurate affect perception in the CMC environment at the significance level of $p=.05$. Daily use and education were non-significant predictor variables for accurate affect perception in the CMC environment ( $p=.56$ and $p=.14$, respectively). Research Hypothesis 2a was supported and Hypothesis $2 \mathrm{~b}$ and Hypothesis $2 \mathrm{c}$ had inconclusive results. Further research focusing on $\mathrm{CMC}$ use and message proficiency may be a valuable project.

Previous research by Tong \& Walther posits that increased language abilities means increased affective interpretation abilities (Joseph B. Walther et al., 2010). Our research did not find evidence to support this claim; however, the combination of affect accuracy prompts and participant education level may be insufficient to fully understand a participant's language ability.

Further research investigating the relationship between demographic variables and affect perception in the CMC environment would be valuable. The present study 
was limited by time and online questionnaire; however, future research may want to focus on the differences between tech natives and tech immigrants. The two groups may be divided up into several more age groups in order to better understand the role of age in affect perception in the CMC environment.

Other areas of interest may want to investigate specific areas of education and affect perception in the $\mathrm{CMC}$ environment. Which disciplines subsequent professions are able to interoperate affect in $\mathrm{CMC}$ with the most accuracy would be interesting research to begin investigating and affective coaching in the professional fields.

\section{Mood and Accurate Affect Perception}

According the research in mood and language, mood is a significant predictor variable in accurate affect perception (Van Berkum et al., 2013). The goal of the present study was to gain further knowledge on the relationship between the positive or negative mood of a message receiver and their accuracy in perceiving the message sender's affect in the CMC environment. Results for Hypothesis 3a was supported while results for Hypothesis $3 b$ was inconclusive.

The multiple linear regression analysis revealed that being in a positive mood significantly improved and individual's ability to accurately interpret affect in the CMC environment at the significance level of $p=.008$. However, being in a negative mood was non-significant at a level of $p=.39$. The results indicate that it is better to read and 
respond to $\mathrm{CMC}$ messages while in a positive mood (over negative mood) due to increase in affect perception accuracy.

Further research on the effects of mood and the perception of affect in the CMC environment may consider focusing on intensity of mood and the relationship it has with the ambiguity of affective words in CMC with the component of time. Our study was limited by one sample of affect per participant. Individuals feel a range of emotions throughout their day. To gain insight into how well an individual reads affect and the relationship mood has on said relationship, asking participants to complete a survey sequence over a period of time would give researchers a range and average score per participant. This techniques would be a more accurate in interpreting an individual's true affect perception capabilities.

\section{Conclusions}

An individual's affect perception influences their subjective and objective experience of their environment (Robinson et al., 2012). The affective experience directly influences cognitions, emotional response, and behaviors (Panksepp, 2003). The present study found that positive mood significantly increases the ability to understand messages in the CMC environment. Learning to cognitively appraise an affective experience is beneficial to social interactions in CMC.

Cognitive appraisal of the stimulus allows time for the affective reaction to move from the lower subcortical regions of the brain to the higher cortical regions where 
complex cognitive reasoning and emotional response can increase the likelihood of positive outcomes of subsequent behaviors (Panksepp, 2003). Cognitive appraisal of affect experiences allow an individual to manipulate CMC to their advantage though gaining insight to their emotional state before replying to a message. As a result, the individual may experience increase in locus of control, overall well-being, and resiliency and a decrease in the effect of daily life stressors (Robinson et al., 2012). Thus, taking the time to cognitively appraise the affective stimulus may lead to a decrease in misunderstanding messages and an increase in positive attitude toward $\mathrm{CMC}$ as a communication medium.

Acknowledgements

Thank you to Chris Allen, Ph.D. and Ann Marie Fallon, Ph.D. for your guidance through the undergraduate and thesis process. It has been a pleasure working with you as your student.

Much appreciation to Todd Bodner, Ph.D. for your assistance on this project and instruction over the past year. You shared your love for data analysis with me, thank you.

To Jeremiah Bullock, M.S., what can I say? Thank you doesn't seem to cover it, but it is a good start. Your assistance, expertise, advice, and patience over this project 
and many other projects has seen me through my entire college experience. Thank you. You are awesome.

To my professors, mentors, and colleagues in Portland State University's Honors College and Psychology Department, thank you for all the time you have spent with me.

Thank you to the Portland State University and the Ford Family for funding my higher education. Without your assistance, none of this would have been possible. 


\section{Works Cited}

Antheunis, M. L., Schouten, A. P., Valkenburg, P. M., \& Peter, J. (2011). Interactive Uncertainty Reduction Strategies and Verbal Affection in Computer-Mediated Communication. Communication Research, 39(6), 757-780.

doi:10.1177/0093650211410420

Bergdall, A. R., Kraft, J. M., Andes, K., Carter, M., Hatfield-Timajchy, K., \& Hock-Long, L. (2012). Love and Hooking Up in the New Millennium: Communication Technology and Relationships among Urban African American and Puerto Rican Young Adults. Journal of Sex Research, 49(6), 570-582. doi:10.1080/00224499.2011.604748

Bohn-Gettler, C. M., \& Rapp, D. N. (2011). Depending on my mood: Mood-driven influences on text comprehension. Journal of Educational Psychology, 103(3), 562-577. doi:10.1037/a0023458

Brown, J. D., \& Taylor, S. E. (1986). Affect and the processing of personal information: Evidence for mood-activated self-schemata. Journal of Experimental Social Psychology, 22(5), 436-452. doi:10.1016/0022-1031(86)90044-2

Burgdorf, J., \& Panksepp, J. (2006). The neurobiology of positive emotions. Neuroscience \& Biobehavioral Reviews, 30(2), 173-187. doi:10.1016/j.neubiorev.2005.06.001

Byron, K. (2008). Carrying Too Heavy a Load? the Communication and Miscommunication of Emotion by Email. Academy of Management Review, 33(2), 309-327. doi:10.5465/AMR.2008.31193163 
Byron, K., \& Baldridge, D. C. (2007). E-Mail Recipients' Impressions of Senders' Likability. Journal of Business Communication, 44(2), 137-160.

Cobb-Clark, D. A., \& Schurer, S. (2012). The stability of big-five personality traits. Economics Letters, 115(1), 11-15. doi:10.1016/j.econlet.2011.11.015

Cohen-Charash, Y., \& Boyd, B. (2007). Mood. In Encyclopedia of industrial and organizational psychology. Thousand Oaks, Calif.: SAGE. Retrieved from http://site.ebrary.com/id/10367425

Cooper, A. J., Smillie, L. D., \& Corr, P. J. (2010). A confirmatory factor analysis of the Mini-IPIP five-factor model personality scale. Personality and Individual Differences, 48(5), 688-691. doi:10.1016/j.paid.2010.01.004

Coyne, S. M., Stockdale, L., Busby, D., Iverson, B., \& Grant, D. M. (2011). “I luv u :)!”: A Descriptive Study of the Media Use of Individuals in Romantic Relationships. Family Relations, 60(2), 150-162. doi:10.1111/j.1741-3729.2010.00639.x

Crawford, J. R. H., Julie D. (2004). The Positive and Negative Affect Schedule (PANAS):Construct validity, measurement properties and normative data in a large non-clinical sample. British Journal of Clinical Psychology, 43(3), 245-265.

Donnellan, M. B., Frederick, L., Oswald, B. M. B., \& Lucas, R. E. (2006). The Mini- IPIP scales: Tiny-yet-effective measures of the Big Five factors of personality. Psychological Assesment, 18, 192-203.

Eysenck, H. J. (1967). The Biological Basis of Personality. Transaction Publishers. Forgas, J. P. (1995). Mood and Judgment: The Affect Infusion Model (AIM). Psychological Bulletin January 1995, 117(1), 39-66. doi:10.1037/0033-2909.117.1.39 
Funder, D. C. (2010). The personality puzzle. New York: W.W. Norton.

Ganong, L. H., Coleman, M., Feistman, R., Jamison, T., \& Stafford Markham, M. (2012). Communication Technology and Postdivorce Coparenting. Family Relations, 61(3), 397-409. doi:10.1111/j.1741-3729.2012.00706.x

Gray, J. A. (1970). The psychophysiological basis of introversion-extraversion. Behaviour Research and Therapy, 8(3), 249-266. doi:10.1016/0005-7967(70)90069-0

Gray, J. A. (1981). A Critique of Eysenck's Theory of Personality. In P. H. J. Eysenck (Ed.), A Model for Personality (pp. 246-276). Springer Berlin Heidelberg. Retrieved from http://link.springer.com/chapter/10.1007/978-3-642-67783-0_8

Hölzel, B. K., Lazar, S. W., Gard, T., Schuman-Olivier, Z., Vago, D. R., \& Ott, U. (2011). How Does Mindfulness Meditation Work? Proposing Mechanisms of Action From a Conceptual and Neural Perspective. Perspectives on Psychological Science, 6(6), 537-559. doi:10.1177/1745691611419671

Howell, R. T., \& Rodzon, K. S. (2011). An exploration of personality-affect relations in daily life: Determining the support for the affect-level and affect-reactivity views. Personality and Individual Differences, 51(7), 797-801. doi:10.1016/j.paid.2011.06.020

Isbell, L. M., \& Burns, K. C. (n.d.). Affect. In Encyclopedia of Social Psychology. 2455 Teller Road, Thousand Oaks California 91320 United States: SAGE Publications, Inc. Retrieved from http://knowledge.sagepub.com/view/socialpsychology/n7.xml 
Kelly, L., \& Keaten, J. A. (2007). Development of the Affect for Communication Channels Scale. Journal of Communication, 57(2), 349-365. doi:10.1111/j.14602466.2007.00346.x

Kwon, S., \& Weed, N. C. (n.d.). Neuroticism. In Encyclopedia of Social Psychology. 2455 Teller Road, Thousand Oaks California 91320 United States: SAGE Publications, Inc. Retrieved from http://knowledge.sagepub.com/view/socialpsychology/n370.xml Larsen, R. J. (n.d.). Independence of Positive and Negative Affect. In Encyclopedia of Social Psychology. 2455 Teller Road, Thousand Oaks California 91320 United States: SAGE Publications, Inc. Retrieved from http://knowledge.sagepub.com/view/socialpsychology/n278.xml

Larsen, R. J., \& Ketelaar, T. (1989). Extraversion, neuroticism and susceptibility to positive and negative mood induction procedures. Personality and Individual Differences, 10(12), 1221-1228. doi:10.1016/0191-8869(89)90233-X

Larsen, R. J., \& Ketelaar, T. (1991). Personality and Susceptibility to Positive and Negative Emotional States. [Miscellaneous Article]. Journal of Personality, 61(1), 132-140.

Lieberman, M. D., \& Rosenthal, R. (2001). Why introverts can't always tell who likes them: Multitasking and nonverbal decoding. Journal of Personality and Social Psychology, 80(2), 294-310. doi:10.1037/0022-3514.80.2.294

Löckenhoff, C. E., \& Costa, P. T. (n.d.). Big Five Personality Traits. In Encyclopedia of Social Psychology. 2455 Teller Road, Thousand Oaks California 91320 United 
States: SAGE Publications, Inc. Retrieved from

http://knowledge.sagepub.com/view/socialpsychology/n67.xml

Lucas, R. E. (n.d.). Extraversion. In Encyclopedia of Social Psychology. 2455 Teller

Road, Thousand Oaks California 91320 United States: SAGE Publications, Inc.

Retrieved from http://knowledge.sagepub.com/view/socialpsychology/n206.xml

Lucas, R. E., \& Baird, B. M. (2004). Extraversion and Emotional Reactivity. [Miscellaneous Article]. Journal of Personality, 86(3), 473-485.

Ochsner, K. N., \& Gross, J. J. (2005). The cognitive control of emotion. Trends in Cognitive Sciences, 9(5), 242-249. doi:10.1016/j.tics.2005.03.010

Odlyzko, A. (2012). Internet Pricing and the History of Communications (SSRN Scholarly Paper No. ID 235283). Rochester, NY: Social Science Research Network. Retrieved from http://papers.ssrn.com/abstract=235283

Panksepp, J. (2003). At the interface of the affective, behavioral, and cognitive neurosciences: Decoding the emotional feelings of the brain. Brain and Cognition, 52(1), 4-14. doi:10.1016/S0278-2626(03)00003-4

Perry, M. S., \& Werner-Wilson, R. J. (2011). Couples and Computer-Mediated Communication: A Closer Look at the Affordances and Use of the Channel. Family and Consumer Sciences Research Journal, 40(2), 120-134. doi:10.1111/j.1552-3934.2011.02099.x

Reisberg, D. (2012). Cognition: Exploring the Science of the Mind (Fifth Edition.). W. W. Norton \& Company. 
Robinson, M. D., Moeller, S. K., Buchholz, M. M., Boyd, R. L., \& Troop-Gordon, W. (2012). The regulatory benefits of high levels of affect perception accuracy: A process analysis of reactions to stressors in daily life. Emotion, 12(4), 785-795. doi:10.1037/a0029044

Russell, J. A. (1980). A circumplex model of affect. Journal of Personality and Social Psychology, 39(6), 1161-1178. doi:10.1037/h0077714

Spitzberg, B. H. (2006). Preliminary Development of a Model and Measure of ComputerMediated Communication (CMC) Competence. Journal of Computer-Mediated Communication, 11(2), 629-666. doi:10.1111/j.1083-6101.2006.00030.x

Suls, J., \& Martin, R. (2005). The Daily Life of the Garden-Variety Neurotic: Reactivity, Stressor Exposure, Mood Spillover, and Maladaptive Coping. Journal of Personality, 73(6), 1485-1510. doi:10.1111/j.1467-6494.2005.00356.x

Sweklej, J., Balas, R., Pochwatko, G., \& Godlewska, M. (2014). Intuitive (in)coherence judgments are guided by processing fluency, mood and affect. Psychological Research, 78(1), 141-149. doi:10.1007/s00426-013-0487-7

Tong, S. T., \& Walther, J. B. (2012). The Confirmation and Disconfirmation of Expectancies in Computer-Mediated Communication. Communication Research. doi:10.1177/0093650212466257

Topi, H., Valacich, J. S., \& Rao, M. T. (2002). The Effects of Personality and Media Differences on the Performance of Dyads Addressing a Cognitive Conflict Task. Small Group Research, 33(6), 667-701. doi:10.1177/1046496402238620 
Uziel, L. (2006). The extraverted and the neurotic glasses are of different colors.

Personality and Individual Differences, 41(4), 745-754.

doi:10.1016/j.paid.2006.03.011

Van Berkum, J. J. A., De Goede, D., Van Alphen, P. M., Mulder, E. R., \& Kerstholt, J. H. (2013). How robust is the language architecture? The case of mood. Frontiers in Psychology, 4. doi:10.3389/fpsyg.2013.00505

Walther, J. B. (1992). Interpersonal Effects in Computer-Mediated Interaction A Relational Perspective. Communication Research, 19(1), 52-90. doi:10.1177/009365092019001003

Walther, J. B. (1996). Computer-Mediated Communication: Impersonal, Interpersonal, and Hyperpersonal Interaction. Communication Research, 23(1), 3-43. doi:10.1177/009365096023001001

Walther, J. B., Deandrea, D. C., \& Tong, S. T. (2010). Computer-Mediated Communication Versus Vocal Communication and the Attenuation of PreInteraction Impressions. Media Psychology, 13(4), 364-386. doi:10.1080/15213269.2010.524913

Watson, D., Clark, L. A., \& Tellegen, A. (1988). Development and validation of brief measures of positive and negative affect: The PANAS scales. Journal of Personality and Social Psychology, 54(6), 1063-1070. doi:10.1037/00223514.54.6.1063

Yuan, J., Zhang, J., Zhou, X., Yang, J., Meng, X., Zhang, Q., \& Li, H. (2012). Neural mechanisms underlying the higher levels of subjective well-being in extraverts: 
Pleasant bias and unpleasant resistance. Cognitive, Affective, \& Behavioral Neuroscience, 12(1), 175-192. doi:10.3758/s13415-011-0064-8 